\title{
Guessing a password over a wireless channel (on the effect of noise non-uniformity)
}

\author{
Mark M. Christiansen and Ken R. Duffy \\ Hamilton Institute \\ National University of Ireland, Maynooth \\ Email: \{mark.christiansen, ken.duffy\}@nuim.ie
}

\author{
Flávio du Pin Calmon and Muriel Médard \\ Research Laboratory of Electronics \\ Massachusetts Institute of Technology \\ Email: \{flavio, medard\}@mit.edu
}

\begin{abstract}
A string is sent over a noisy channel that erases some of its characters. Knowing the statistical properties of the string's source and which characters were erased, a listener that is equipped with an ability to test the veracity of a string, one string at a time, wishes to fill in the missing pieces. Here we characterize the influence of the stochastic properties of both the string's source and the noise on the channel on the distribution of the number of attempts required to identify the string, its guesswork. In particular, we establish that the average noise on the channel is not a determining factor for the average guesswork and illustrate simple settings where one recipient with, on average, a better channel than another recipient, has higher average guesswork. These results stand in contrast to those for the capacity of wiretap channels and suggest the use of techniques such as friendly jamming with pseudo-random sequences to exploit this guesswork behavior.
\end{abstract}

\section{INTRODUCTION}

[

This paper quantifies the influence of the stochastic properties of a string source and of a noisy erasure channel on the difficulty a listener has in guessing the erased pieces of the string. As a concrete example in advance of the mathematical abstraction, consider a proximity card reader where an electronic signature, a password, is wirelessly transmitted when the card is near the reader. An unintended recipient is eavesdropping, but overhears the card's transmission via a noisy channel that erases certain characters. If the eavesdropper knows the string's source statistics and which characters were erased, how many guesses must he make before identifying the one that causes the card reader to notify success?

For i.i.d. character sources and noise that is independent of the string, but possibly correlated, Theorem 1 answers this question, providing an asymptotic approximation to the guesswork distribution as the string becomes long. Corollary 1 establishes that the mean number of erasures on the channel and the Shannon entropy of the character source determine the growth rate of the expected logarithm of the number of guesses required to identify the erased sub-string. The exponential growth rate of the average number of guesses, however, is

F.d.P.C. and M.M. sponsored by the Department of Defense under Air Force Contract FA8721-05-C-0002. Opinions, interpretations, recommendations, and conclusions are those of the authors and are not necessarily endorsed by the United States Government. Specifically, this work was supported by Information Systems of ASD(R\&E). determined by the scaling of the asymptotic moment of the number of erasures evaluated at the Rényi entropy, with parameter $1 / 2$, of the character distribution.

As a consequence of these results, we provide examples illustrating that the average guesswork can be smaller on a channel that is, on average, noisier demonstrating that average noise is not a useful statistic for guesswork. This conclusion may seem counterintuitive in the context of capacity results for Wyner's wire-tap [1] that, when applied to an erasure channel, indicate that secrecy capacity is non-zero only if the probability of erasure of the intended party is lower than that of the eavesdropper. Results in which a first receiver, with more erasures (on average) than a second receiver, can better recover a message than the second receiver are, to the authors' knowledge, few. One recent exception is [2], which also considers the effect of randomness of erasures in message recovery. In contrast to our work, the authors consider secret message capacity in a specific setting that uses feedback to provide causal channel state information for the intended receiver, allowing the sender to transmit in a way that is advantageous to the intended receiver. In the case of two parties with an erasure, their scheme relies on the fact that the secret key agreement by public discussion from common information developed by [3] reduces to requiring only the channel state be shared over a public channel.

Guesswork analysis of a distinct wiretap model to the one considered here is provided in [4], [5]. There it is assumed that an eavesdropper observes a string that has been encrypted with a function employing a uniformly chosen random key. The impact of key rate on asymptotic moments of guessing is determined.

\section{GUESSWORK AND ERASURE CHANNELS}

We begin with summarizing material on the mathematical formulation for guesswork followed by a brief overview of the relevance of erasure channels as models of wireless communication. Let $\mathbb{A}=\{0, \ldots, m-1\}$ be a finite alphabet and consider a stochastic sequence of words, $\left\{W_{k}\right\}$, where $W_{k}$ is a string of length $k$ taking values in $\mathbb{A}^{k}$. Assume that a word is selected and an inquisitor is equipped with a device, such as a one-way hash function, through which a word can be tested one at a time. With no information beyond the string length 
and the source statistics, their optimal strategy to identify the word is to generate a partial-order of the words from most likely to least likely and guess them in turn. That is, for each $k$ the attacker generates a function $G: \mathbb{A}^{k} \rightarrow\left\{1, \ldots, m^{k}\right\}$ such that $G\left(w^{\prime}\right)<G(w)$ if $P\left(W_{k}=w^{\prime}\right)>P\left(W_{k}=w\right)$. For a word $w$ the integer $G(w)$ is the number of guesses until the string $w$ is guessed, its Guesswork.

Massey [6] established that the Shannon entropy of the word source bears little relation to the average guesswork. As word length grows an asymptotic relationship between scaled moments of the guesswork distribution and specific Rényi entropy was identified under weak assumptions on the stochastic properties of the string source [7], [8], [9], [10]. These results have recently been built upon to establish that $\left\{k^{-1} \log G\left(W_{k}\right)\right\}$ satisfies a Large Deviation Principle (LDP) [11], giving a direct approximation to the guesswork distribution, $P\left(G\left(W_{k}\right)=n\right)$ for $n \in\left\{1, \ldots, m^{k}\right\}$.

In the present article we restrict to i.i.d. letter sources, but include noise sources that could potentially be correlated. This enables us to consider the erasures as a subordinating process for the guesswork, as will become clear.

Assumption 1: The string $W_{k}$ is constituted of independent and identically distributed characters with distribution $P\left(W_{1}=i\right)$ for $i \in \mathbb{A}$.

Under this assumption, if one must guess the entire word $W_{k}$, the following result is known.

Proposition 1 ([7], [9], [11]): The scaled Cumulant Generating Function (sCGF) of $\left\{k^{-1} \log G\left(W_{k}\right)\right\}$ exists

$$
\begin{aligned}
\Lambda_{G}(\alpha) & =\lim _{k \rightarrow \infty} \frac{1}{k} \log E\left(\exp \left(\alpha \log \left(G\left(W_{k}\right)\right)\right)\right) \\
& = \begin{cases}\alpha R\left(\frac{1}{1+\alpha}\right) & \text { if } \alpha>-1 \\
-R(\infty) & \text { if } \alpha \leq-1,\end{cases}
\end{aligned}
$$

where $R(\alpha)$ is the Rényi entropy with parameter $\alpha$,

$$
\begin{aligned}
R(\alpha) & =\frac{1}{1-\alpha} \log \left(\sum_{i \in \mathbb{A}} P\left(W_{1}=i\right)^{\alpha}\right) \\
R(\infty) & =-\max _{i \in \mathbb{A}} \log P\left(W_{1}=i\right) .
\end{aligned}
$$

Moreover, the process $\left\{k^{-1} \log G\left(W_{k}\right)\right\}$ satisfies a Large Deviation Principle with rate function

$$
\Lambda_{G}^{*}(x)=\sup _{\alpha \in \mathbb{R}}\left(x \alpha-\Lambda_{G}(\alpha)\right) .
$$

As in [7], setting $\alpha=1$ equation (1) gives

$$
\begin{aligned}
\Lambda_{G}(1) & =\lim _{k \rightarrow \infty} \frac{1}{k} \log E\left(G\left(W_{k}\right)\right) \\
& =R(1 / 2)=2 \log \left(\sum_{i \in \mathbb{A}} P\left(W_{1}=i\right)^{1 / 2}\right),
\end{aligned}
$$

establishing that the exponential growth rate of the average guesswork as the string gets longer is governed by Rényi entropy of the character distribution with parameter $1 / 2$, which is greater than its Shannon entropy, with equality if and only if the character source is uniformly distributed. The
LDP gives the following approximation [11] for large $k$ and $n \in\left\{1, \ldots, m^{k}\right\}$,

$$
P\left(G\left(W_{k}\right)=n\right) \approx \frac{1}{n} \exp \left(-k \Lambda_{G} *\left(\frac{1}{k} \log n\right)\right) .
$$

Erasure models are common for coded communications. They arise for systems where an underlying error-correcting code can fail to correct the errors, but error-detection mechanisms will lead to detection of the failure to correct. While it is possible for errors to remain uncorrected in such a way that the receiver cannot detect the failure to correct. That traditional algebraic codes with $n$ symbols of redundancy can correct up to $n$ errors but detect up to $2 n-1$ errors justifies the common assumption that failures to detect errors may be neglected, whereas failures to correct may not. Failure to correct errors may be a design goal in certain systems. In wiretap channels, codes are deliberately constructed in such a way that, under channel conditions less favorable than those of the intended receiver, codes fail to decode (e.g. [12]).

\section{Subordinated Guesswork - General Results}

We wish to consider the guesswork required to identify a string, $W_{k}$, sent over a stochastic, noisy channel that erases characters. We assume that a listener is equipped with an ability to test the veracity of each missing sub-string and wishes to fill in the missing piece. As the string $W_{k}$ is made up of i.i.d. characters, if $N_{k} \in\{1, \ldots, k\}$ is the number of characters erased by the noise, the listener must effectively guess a word of $N_{k}$ characters in length. Thus we are interested in properties of the the guesswork of the word subordinated by the erasures process, $G\left(W_{N_{k}}\right)$, wishing to understand the influence of the properties of the string source and the noise on the channel on the distribution of the number of attempts required to identify the missing sub-string.

While we assume that the string is made up of i.i.d. characters, the noise process can be correlated and we make the following assumption, which encompasses, for example, Markovian erasure processes.

Assumption 2: The noise process is such that $\left\{N_{k} / k\right\}$, where $N_{k}$ is the number of erasures due to noise in a string of length $k$, satisfies a LDP with convex rate function $\Lambda_{N}{ }^{*}: \mathbb{R} \mapsto[0, \infty]$ such that $\Lambda_{N}{ }^{*}(y)=\infty$ if $y \notin[0,1]$.

Loosely speaking, assumption 2 implies that $P\left(N_{k} \approx y k\right) \asymp$ $\exp \left(-k \Lambda_{N}{ }^{*}(y)\right)$. Our main general, structural result is the following.

Theorem 1: The subordinated guesswork process $\left\{1 / k \log G\left(W_{N_{k}}\right)\right\} \quad$ satisfies a LDP with convex rate function

$$
\Lambda_{N G}^{*}(x)=\inf _{y \in[0,1]}\left(y \Lambda_{G}{ }^{*}\left(\frac{x}{y}\right)+\Lambda_{N}{ }^{*}(y)\right) .
$$

The sCGF for $\left\{1 / k \log G\left(W_{N_{k}}\right)\right\}$, the Legendre-Fenchel transform of $\Lambda_{N G}^{*}$, is given by the composition of the sCGF for the noise with SCGF for the non-subordinated guesswork

$$
\begin{aligned}
\Lambda_{N G}(\alpha) & =\lim _{k \rightarrow \infty} \frac{1}{k} \log E\left(\exp \left(\alpha \log \left(G\left(W_{N_{k}}\right)\right)\right)\right) \\
& =\Lambda_{N}\left(\Lambda_{G}(\alpha)\right) .
\end{aligned}
$$


Proof: The method of proof of the LDP is akin to that used in [11], establishing that the upper and lower deviation functions coincide, followed by an application of the contraction principle. With $B_{\epsilon}(x)=(x-\epsilon, x+\epsilon)$. We first show that

$$
\begin{aligned}
& \lim _{\epsilon \downarrow 0} \liminf _{k \rightarrow \infty} \frac{1}{k} \log P\left(\frac{1}{k} \log G\left(W_{N_{k}}\right) \in B_{\epsilon}(x), \frac{N_{k}}{k} \in B_{\epsilon}(y)\right) \\
& =\lim _{\epsilon \downarrow 0} \limsup _{k \rightarrow \infty} \frac{1}{k} \log P\left(\frac{1}{k} \log G\left(W_{N_{k}}\right) \in B_{\epsilon}(x), \frac{N_{k}}{k} \in B_{\epsilon}(y)\right) \\
& =y \Lambda_{G}{ }^{*}\left(\frac{x}{y}\right)+\Lambda_{N}{ }^{*}(y) \text { for all } x \geq 0, y \in[0,1] .
\end{aligned}
$$

For example, for $y \in(0,1]$, consider

$$
\begin{aligned}
& \frac{1}{k} \log P\left(\frac{1}{k} \log G\left(W_{N_{k}}\right) \in B_{\epsilon}(x), \frac{N_{k}}{k} \in B_{\epsilon}(y)\right) \\
\geq & \frac{1}{k} \log P\left(\frac{1}{k} \log G\left(W_{\lfloor k(y-\epsilon)\rfloor}\right) \in B_{\epsilon}(x)\right) \\
& +\frac{1}{k} \log P\left(\frac{N_{k}}{k} \in B_{\epsilon}(y)\right) .
\end{aligned}
$$

Taking $\liminf \operatorname{in}_{k \rightarrow \infty}$, using the LDPs for $\left\{k^{-1} \log G\left(W_{k}\right)\right\}$ and $\left\{N_{k} / k\right\}$ followed by $\lim _{\epsilon \downarrow}$ gives an appropriate lower bound. An equivalent upper bound follows similarly.

For $y=0$, if $x>0$ we can readily show that the upper deviation function takes the value $-\infty$ as $G\left(W_{\lfloor\epsilon y\rfloor}\right) \leq m^{y \epsilon}$. If $x=0$, then the limsup bound is achieved by solely considering the noise term, while for the liminf consider the ball $G\left(W_{N_{k}}\right) \leq \exp (k \epsilon \log (m))$, which has probability 1 and so the upper and lower deviation functions again coincide.

As the state space is compact, the LDP for $\left\{\left(1 / k \log G\left(W_{N_{k}}\right), N_{k} / k\right)\right\}$ follows (e.g. [13], [14]) with the rate function $y \Lambda_{G}{ }^{*}(x / y)+\Lambda_{N}{ }^{*}(y)$. From this LDP, the LDP for $\left\{\left(1 / k \log G\left(W_{N_{k}}\right)\right\}\right.$ via the contraction principle [14] by projection onto the first co-ordinate.

To prove that $\Lambda_{N G}^{*}(x)$ is convex in $x$, first note that $y \Lambda_{G}{ }^{*}(x / y)$ is jointly convex in $x$ and $y$, with $y>0$, by the following argument. For $\beta \in(0,1)$, set $\eta=\beta y_{1} /\left(\beta y_{1}+\right.$ $\left.(1-\beta) y_{2}\right) \in[0,1]$ and note that

$$
\begin{aligned}
& \left(\beta y_{1}+(1-\beta) y_{2}\right) \Lambda_{G} *\left(\frac{\beta x_{1}+(1-\beta) x_{2}}{\beta y_{1}+(1-\beta) y_{2}}\right) \\
& =\left(\beta y_{1}+(1-\beta) y_{2}\right) \Lambda_{G} *\left(\eta \frac{x_{1}}{y_{1}}+(1-\eta) x_{2} y_{2}\right) \\
& \leq \beta y_{1} \Lambda_{G}^{*}\left(\frac{x_{1}}{y_{1}}\right)+(1-\beta) y_{2} \Lambda_{G}^{*}\left(\frac{x_{2}}{y_{2}}\right)
\end{aligned}
$$

where we have used the convexity of $\Lambda_{G}{ }^{*}$. As the sum of convex functions is convex, $y \Lambda_{G}{ }^{*}(x / y)+\Lambda_{N}{ }^{*}(y)$ is convex and as the point-wise minimum of a jointly convex function is convex, $\Lambda_{N G}^{*}(x)$ is convex.

An application of Varadhan's Lemma (Theorem 4.3.1 [14]) identifies the sCGF for the subordinated process as the Legendre Fenchel transform of $\Lambda_{N G}^{*}$, $\sup _{x \in \mathbb{R}}\left(\alpha x-\Lambda_{N G}^{*}(x)\right)$. To convert this into an expression in terms of $\Lambda_{N}$ and $\Lambda_{G}$ observe that

$$
\begin{aligned}
\sup _{x \in \mathbb{R}}\left(\alpha x-\Lambda_{N G}^{*}(x)\right) & =\sup _{x \in \mathbb{R}} \sup _{y \in \mathbb{R}}\left(\alpha x-y \Lambda_{G}{ }^{*}\left(\frac{x}{y}\right)-\Lambda_{N}^{*}(y)\right) \\
& =\sup _{y \in \mathbb{R}}\left(y \sup _{z \in \mathbb{R}}\left(\alpha z-\Lambda_{G}{ }^{*}(z)\right)-\Lambda_{N}{ }^{*}(y)\right) \\
& =\sup _{y \in \mathbb{R}}\left(y \Lambda_{G}(\alpha)-\Lambda_{N}{ }^{*}(y)\right) \\
& =\Lambda_{N}\left(\Lambda_{G}(\alpha)\right) .
\end{aligned}
$$

Theorem 11, in particular, identifies the growth rate of the average subordinated guesswork.

Corollary 1: The growth rate of the average of the logarithm of the subordinated guesswork is determined by the average noise and the Shannon entropy of the character source

$$
\begin{aligned}
\lim _{k \rightarrow \infty} \frac{1}{k} E\left(\log G\left(W_{N_{k}}\right)\right) & =\left.\frac{d}{d \alpha} \Lambda_{N}\left(\Lambda_{G}(\alpha)\right)\right|_{\alpha=0} \\
& =\mu_{N} H_{G},
\end{aligned}
$$

where

$\mu_{N}=\lim _{k \rightarrow \infty} \frac{E\left(N_{k}\right)}{k}, H_{G}=-\sum_{i \in \mathbb{A}} P\left(W_{1}=i\right) \log P\left(W_{1}=i\right)$.

The growth rate of the average subordinated guesswork is, however, given by the sCGF of the noise evaluated at the character Rényi entropy at $1 / 2$,

$$
\begin{aligned}
\lim _{k \rightarrow \infty} \frac{1}{k} \log E\left(G\left(W_{N_{k}}\right)\right) & =\Lambda_{N}\left(\Lambda_{G}(1)\right) \\
& =\lim _{k \rightarrow \infty} \frac{1}{k} \log E\left(\exp \left(R(1 / 2) N_{k}\right)\right) .
\end{aligned}
$$

Thus the determining factor in the average guesswork is not the average noise, but the scaling of the cumulant of the noise process determined by the Rényi entropy with parameter $1 / 2$.

\section{EXAMPLES}

Corollary 1 suggests the design of schemes whose principle is to ensure that the stochastic properties of either the the noise or the source, as manifested through the behavior of $\Lambda_{N}\left(\Lambda_{G}(1)\right)$, differs between intended receivers and unintended receivers so as to provide a lower growth rate in the average subordinated guesswork of intended receivers. The intended and unintended receivers may observe the transmitted string through parallel channels or through a common channel where there exists a dependence in the noise at different receivers. Such scenarios mirror those that have been considered for secrecy capacity, with the latter having been extensively studied as a model for wireless channels in which the unintended receivers are eavesdroppers (e.g. [12]) and the former considered less often [15], [16], [17].

In order to reduce the guesswork of intended receivers over eavesdroppers, common randomness between the word subordinated by the erasures process and the intended receivers may be used as common randomness is a means of generating a secret key [18], [3], [19]. Common randomness for secrecy may be derived from the source itself, for instance as side 
information regarding the source. Channel side information is commonly proposed or used as a source of common randomness in wireless networks (e.g. [20] and references therein). The use of differentiated channel side information for guesswork in erasure channels provides a means of tuning properties of the sCGF of the noise, $\Lambda_{N}$.

In wireless erasure channels, we may consider several means of achieving differentiated channel side information between intended receivers and eavesdroppers. Consider, for example, a fading channel, where fades lead to erasures and where fading characteristics permit prediction of future fades from current channel measurements. A node that actively sounds the channel, or receives channel side information from a helper node, may know, perfectly or imperfectly, which erasures will occur over some future time.

Friendly jamming instantiates different channel side information between intended and unintended receivers by actively modifying the channel. Friendly jamming have been proposed and demonstrated to modify secrecy regions in wiretap-like settings [21], [22]. A notion related to friendly jamming is that of cooperative jamming [23] where multiple users collude in their use of the shared channel in order to reduce an eavesdropper's ability. In our setting, a jammer using a pseudo-noise sequence that is known to the receiver but appearing Bernoulli to the eavesdropper can render the average guesswork of the intended receiver to be lower than that of the eavesdropper. Such friendly jamming may be effective even if the jammer generates, on average, more erasures for the intended receiver than for the eavesdropper, for instance because it may be closer to the former than to the latter. The same mechanism can be used to create attacks in which a jammer, using a sequence known to the eavesdropper but not to the receiver, may increase the guesswork of the intended receiver relatively to that of the receiver.

We explore numerically the effect of modifying the distribution of the source or erasures, for instance through the use of friendly jamming. Corollary 1 makes clear that the growth rate of the average subordinated guesswork depends upon unusual statistics of both the noise and of the character source. We illustrate the ramifications of this by demonstrating that an unintended eavesdropper can have a better channel, on average, yet have a larger average guesswork than an intended recipient with a noisier average channel. We illustrate this in two ways: with the character distribution fixed and varying the noise, and vice versa.

Consider the simplest example, where an unintended recipient has an i.i.d. channel with a probability of erasure of $p$ per character. This is a typical channel model and gives

$$
\Lambda_{N}(\beta)=\log \left(1-p+p e^{\beta}\right) .
$$

Thus his average subordinated guesswork growth rate is

$$
\Lambda_{N}(R(1 / 2))=\log \left(1-p+p e^{R(1 / 2)}\right) \geq p R(1 / 2),
$$

where the latter follows by Jensen's inequality and unless $p=$ 0 or 1 the inequality is strict.

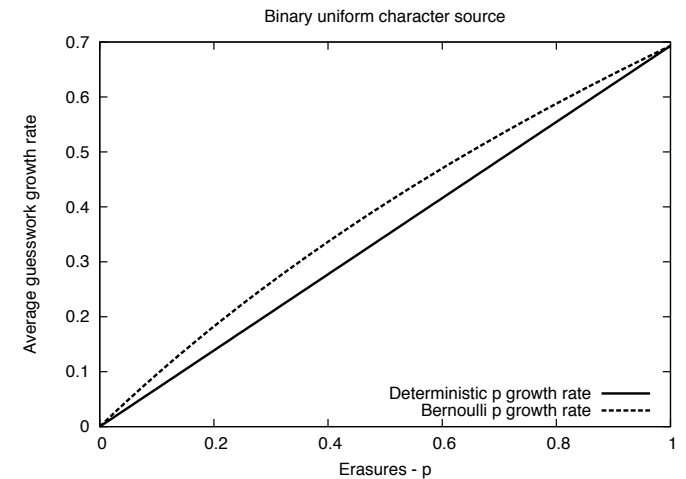

Fig. 1. Binary source alphabet, $\mathbb{A}=\{1,2\}$, with $P\left(W_{1}=1\right)=1 / 2$. Average guesswork growth rate for deterministic channel with proportion $p$ characters erased compared to a memoryless Bernoulli $p$ erasure channel. For a given average number of erasures, the deterministic channel has a lower average guesswork.

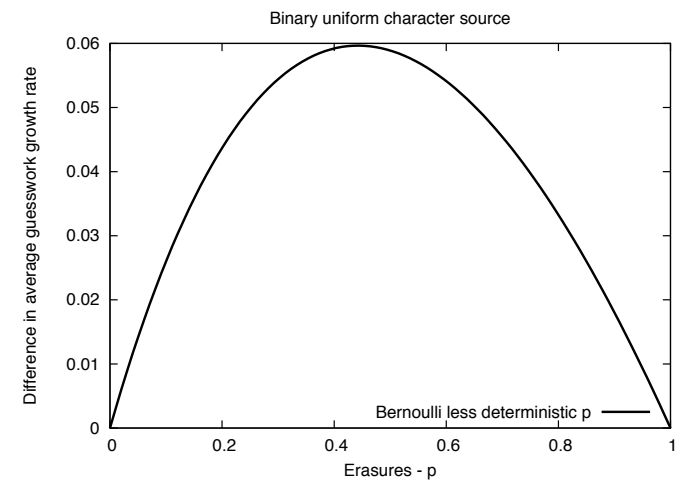

Fig. 2. Binary source alphabet, $\mathbb{A}=\{1,2\}$, with $P\left(W_{1}=1\right)=1 / 2$ Similar to Figure 1 but plotting the difference between the Bernoulli $p$ average guesswork growth rate and the deterministic $p$ average guesswork.

If the intended receiver has a deterministic channel with a proportion $\mu$ of characters erased, then the growth rate of its average subordinated guesswork is $\mu R(1 / 2)$. In particular, if $p<\mu<R(1 / 2)^{-1} \log (1-p+p \exp (R(1 / 2)))$ then even though the channel of the unintended recipient is, on average, more noisy than the intended recipient, the average guesswork of the latter is smaller.

Assuming a binary alphabet, $\mathbb{A}=\{1,2\}$, we present three figures to illustrate that the average guesswork depends upon both the channel and source statistics. First, fix the source statistics by assuming $P\left(W_{1}=1\right)=1 / 2$. For $p \in[0,1]$, Figure 11 plots the average guesswork growth rate for the deterministic channel $p R(1 / 2)$ and for the Bernoulli channel $\log (1-p+p \exp (R(1 / 2)))$. If $p \neq 0$ or 1 , the Bernoulli channel has a higher average guesswork. Thus the intended recipient could have, on average, a less noisy channel, yet have a lower average guesswork. For clarity, Figure 2 plots the difference between these growth rates.

Figures 1 and 2 highlight the influence of the channel statistics on the average guesswork growth rate, but Figure 3 demonstrates the confounding influence of the source statistics. Here we assume that one channel is deterministic with $14 \%$ of characters erased while the other channel is Bernoulli with an average of $10 \%$ characters erased. Figure 3 plots the difference 


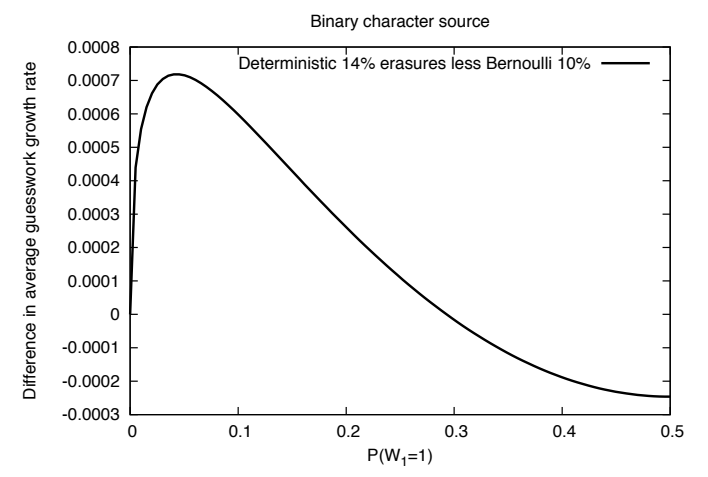

Fig. 3. Binary source alphabet, $\mathbb{A}=\{1,2\}$. Difference in average guesswork growth rate, as a function of $P\left(W_{1}=1\right)$, between a deterministic channel with $14 \%$ characters erased and a Bernoulli channel with $10 \%$ chance that each character is deleted. If the character source is less variable, the deterministic channel has a higher growth rate, but as the character source becomes more variable, it has a lower growth rate.

in average guesswork growth rate between these two channels as the source statistics change. If the source is less variable, the deterministic channel has a higher average guesswork, but as the source statistics become more variable, this reverses and the Bernoulli channel has higher average guesswork growth rate. In other words, even though the average noise on the deterministic channel is worse, dependent upon the source statistics its average guesswork may be lower than a Bernoulli channel with lower average noise.

Between them, these examples indicate the trade-off in influence of the source and noise statistics on the guesswork. While we have assumed the simplest noise channels, these results are characteristic of the system.

\section{CONCLUSIONS}

We have characterized the asymptotic distribution of the guesswork required to reconstitute a string that has been subject to symbol erasure, as occurs on noisy communication channels. The scaled Cumulant Generating Function of the guesswork subordinated by the erasure process has a simple characterization as the composition of the SCGF of the noise with the sCGF of the unsubordinated guesswork. This form is redolent of the well-known result for the moment generating function for a random sun of random summands, but is an asymptotic result for guesswork. These results suggest that methods inspired from the secrecy capacity literature, such as the use of differentiated channel or source side information between the intended receiver and the eavesdropper, can be used in the context of guesswork. Indeed, numerical examples show that deterministic erasures can lead to lower average guesswork than Bernoulli erasures with a lower mean number of erasures. In further work, one may consider the behavior of guesswork in different settings that have been explored in the wiretap and cognate literature.

One may also envisage generalizing this analysis to the case where there are retransmissions of the entire string or of the symbols that have not been received by the intended receiver. Retransmissions are commonly employed in several protocols to enable reliability and, in the case of an erasure channel with perfect feedback, taking the form of acknowledgments, uncoded retransmission is capacity-achieving.

\section{REFERENCES}

[1] A. D. Wyner, "The wire-tap channel," Bell Syst. Tech. J., vol. 54, no. 8, pp. 1355-1387, 1975.

[2] L. Czap, V. M. Prabhakaran, C. Fragouli, and S. Diggavi, "Secret message capacity of erasure broadcast secret message capacity of erasure broadcast channels with feedback," in Information Theory Workshop, 2011.

[3] U. Maurer, "Secret key agreement by public discussion from common information," IEEE Trans. Inf. Theory, vol. 39, no. 3, pp. 733-742, 1993.

[4] N. Merhav and E. Arikan, "The Shannon cipher system with a guessing wiretapper," IEEE Trans. Inform. Theory, vol. 45, no. 6, pp. 1860-1866, 1999.

[5] M. K. Hanawal and R. Sundaresan, "The Shannon cipher system with a guessing wiretapper: General sources," IEEE Trans. Inform. Theory, vol. 57, no. 4, pp. 2503-2516, 2011.

[6] J. L. Massey, "Guessing and entropy," IEEE Int. Symp. Inf Theory, pp. 204-204, 1994.

[7] E. Arikan, "An inequality on guessing and its application to sequential decoding," IEEE Trans, Inf. Theory, vol. 42, no. 1, pp. 99-105, 1996.

[8] D. Malone and W. Sullivan, "Guesswork and entropy," IEEE Trans. Inf. Theory, vol. 50, no. 4, pp. 525-526, 2004.

[9] C.-E. Pfister and W. Sullivan, "Rényi entropy, guesswork moments and large deviations," IEEE Trans. Inf. Theory, no. 11, pp. 2794-00, 2004.

[10] M. K. Hanawal and R. Sundaresan, "Guessing revisited: A large deviations approach," IEEE Trans. Inf. Theory, vol. 57, no. 1, pp. 70-78, 2011.

[11] M. M. Christiansen and K. R. Duffy, "Guesswork, large deviations and Shannon entropy," IEEE Trans. Inf. Theory, vol. 59, no. 2, pp. 796-802, 2013.

[12] M. Bloch and J. Barros, Physical-Layer Security: From Information Theory to Security Engineering. Cambridge Press, 2011.

[13] J. T. Lewis and C.-E. Pfister, "Thermodynamic probability theory: some aspects of large deviations," Russian Math. Surveys, vol. 50, no. 2, pp 279-317, 1995.

[14] A. Dembo and O. Zeitouni, Large Deviations Techniques and Applications. Springer-Verlag, 1998.

[15] H. Yamamoto, "On secret sharing communication systems with two or three channels," IEEE Trans. Inf. Theory, vol. 32, no. 3, pp. 387-393, 1986.

[16] $ـ$, "A coding theorem for secret sharing communication systems with two Gaussian wiretap channels," IEEE Trans. Inf. Theory, vol. 37, no. 3, pp. 634-638, 1991.

[17] L. Zang, R. Yates, and W. Trappe, "Secrecy capacity of independent parallel channels," in Allerton Conference on Communicatoin, Control and Computation, 2006.

[18] R. Ahslwede and I. Csiszár, "Common randomness in information theory and cryptography-part I: Secret sharing," IEEE Trans. Inf. Theory, vol. 39, no. 4, pp. 1121-1132, 1993.

[19] I. Csiszár and P. Narayan, "Common randomness and secret key generation with a helper," IEEE Trans. Inf. Theory, vol. 46, no. 2, pp. 344-366, 2000.

[20] S. Mathur, R. Miller, W. Trappe, N. Mandayam, and A. Varshavsky, "Clique: Proximity-based secure pairing of wireless devices," in Proceedings of ACM CoNEXT, 2010.

[21] J. P. Vilela, M. Bloch, J. Barros, and S. W. McLaughlin, "Wireless secrecy regions with friendly jamming," IEEE Trans. Inf. Forensics Security, vol. 6, no. 2, pp. 256-266, 2011.

[22] S. Gollakota, H. Hassanien, B. Ransford, D. Katabi, and K. Fu, "They can hear your heartbeats: Non-invasive security for implantable medical device," in Sigcomm, 2011.

[23] E. Tekin and A. Yener, "The general Gaussian multiple-access and twoway wiretap channels: Achievable rates and cooperative jamming," IEEE Trans. Inf. Theory, vol. 54, no. 6, pp. 2735-2751, 2008 